\title{
Pentatomidade (Hemiptera: Heteroptera) of the Pampa biome: Serra do Sudeste and Parque de Espinilho da Barra do Quaraí, Rio Grande do Sul, Brazil
}

\author{
Paulo Roberto Sousa Bunde ${ }^{1}$,Jocélia Grazia ${ }^{1,2,7}$, Milton de Souza Mendonça Junior ${ }^{1,3}$, \\ Cristiano Feldens Schwertner ${ }^{1,4}$, Eduardo José Ely e Silva ${ }^{5}$ \& Élen Nunes Garcia ${ }^{6}$ \\ ${ }^{1}$ Programa de Pós-graduação em Biologia Animal, Instituto de Biociências, \\ Universidade Federal do Rio Grande do Sul - UFRGS, \\ Av. Bento Gonçalves, 9500, Bloco IV-Prédio, CP 434345, CEP 91501-970, \\ Porto Alegre, RS, Brasil,e-mail: paulobunde@ig.com.br \\ ${ }^{2}$ Departamento de Zoologia, Universidade Federal do Rio Grande do Sul - UFRGS \\ ${ }^{3}$ Departamento de Ecologia, Universidade Federal do Rio Grande do Sul-UFRGS, \\ e-mail:milton.mendonca@ufrgs.br \\ ${ }^{4}$ Departamento de Ciências Biológicas, Universidade Federal de São Paulo - UNIFESP, Campus Diadema, \\ Rua Artur Riedel, 275, Eldorado, CEP 09972-270, Diadema, SP, Brasil, e-mail: acrosternum@yahoo.com.br \\ ${ }^{5}$ Departamento de Fitossanidade, Faculdade de Agronomia Eliseu Maciel, \\ Universidade Federal de Pelotas - UFPel, Campus Universitário, e-mail: eduelysilva@uol.com.br \\ ${ }^{6}$ Departamento de Botânica, Instituto de Biologia, Universidade Federal de Pelotas - UFPel, \\ e-mail: elengarcia@uol.com.br \\ ${ }^{7}$ Corresponding author: Jocelia Grazia,e-mail: jocelia@ufrgs.br
}

BUNDE, P.R.S., GRAZIA, J., MENDONÇA JÚNIOR, M.S., SCHWERTNER, C.F., SILVA, E.J.E. \& GARCIA, É.N. Pentatomidade (Hemiptera: Heteroptera) of the Pampa biome: Serra do Sudeste and Parque de Espinilho da Barra do Quaraí, Rio Grande do Sul, Brazil. Biota Neotrop., 10(3): http://www.biotaneotropica. org.br/v10n3/en/abstract?article+bn01310032010.

\begin{abstract}
A list of the Pentatomidae species of the Pampa biome, State of Rio Grande do Sul, Brazil is provided. The list is based on surveys carried out in the Serra do Sudeste, Canguçu and Caçapava counties, and in Parque de Espinilho da Barra do Quaraí during 2003-2004; these areas were never collected in Rio Grande do Sul state before. Sweeping net and beating tray were used to sample herb layer, and trees and shrubs, respectively. Fifty one species of Pentatomidae were registered, distributed in five subfamilies (Asopinae, Cyrtocorinae, Discocephalinae, Edessinae and Pentatominae) and 27 genera. The Pentatominae subfamily had the largest number of species (32 spp.). All the registered localities represent new records to the local fauna, and host plants were identified for nine pentatomid species.
\end{abstract}

Keywords: stink bugs, heteropterous insects, inventory, South of Brazil.

BUNDE, P.R.S., GRAZIA, J., MENDONÇA JUNIOR, M.S., SCHWERTNER, C.F., SILVA, E.J.E. \& GARCIA, É.N. Pentatomidade (Hemiptera: Heteroptera) do bioma Pampa: Serra do Sudeste e Parque Estadual do Espinilho, Rio Grande do Sul, Brasil. Biota Neotrop. 10(3): http://www.biotaneotropica.org.br/v10n3/pt/ abstract?article+bn01310032010.

Resumo: Uma lista das espécies de Pentatomidae do bioma Pampa, Estado do Rio Grande do Sul, Brasil, é aqui apresentada. Esta lista teve como base as expedições de coleta realizadas na Serra do Sudeste, nos municípios de Canguçu e Caçapava, e no Parque Estadual do Espinilho durante os anos de 2003-2004; estas áreas nunca foram antes amostradas no Rio Grande do Sul. Cinquenta e uma espécies de Pentatomidae foram registradas, distribuídas em cinco subfamílias (Asopinae, Cyrtocorinae, Discocephalinae, Edessinae and Pentatominae) e 27 gêneros. A subfamília Pentatominae apresentou o maior número de espécies (32 spp.). Todas as localidades em que foram coletados os insetos representam novos registros para a fauna local e nove espécies de Pentatomidae tiveram suas plantas hospedeiras registradas.

Palavras-Chave: percevejos-do-mato, heterópteros, inventário, Sul do Brasil. 


\section{Introduction}

Species lists are basic tools to the knowledge of the biological diversity. They also help to monitor the changes in the components of this diversity due to environmental impacts, either natural or caused by human activities (Lewinsohn et al. 2001). Besides, species lists are indispensable to taxonomic and biogeographic studies.

Very little is known about the fauna of the Pampa region overall (Levinsohn \& Prado 2002, MMA 2007a), especially for invertebrates. This is considered the main barrier to the conservation of this region. Almost every year new species are described (i.e. Verrastro et al. 2003, Bunde et al. 2006, Ferrer et al. 2008), however some areas are still totally unknown (MMA 2007a). Aguiar et al. (2009), aiming to provide guidelines for the study of the Entomodiversity in Brazil, stressed the necessity of surveys in less investigated areas and habitats; one of those areas was the Pampa biome: Serra do Sudeste and Campos sulinos.

The Pentatomidae family is one of the 29 families of the infraorder Pentatomomorpha (Schuh \& Slater 1995). This family is well represented in all biogeographical regions, including more than 4,500 species. About 650 species are estimated to occur in Brazil (Grazia et al. in press). The phytophagous habit is the ancestral condition in the family; the majority of the species feed on different parts of the host plants, specially seeds, fruits and flowers (Schuh \& Slater 1995, Grazia \& Schwertner 2008). Among these phytophagous species, some are considered crop pests (Panizzi et al. 2000). On the other hand, the predator habit evolved secondarily at least once within Pentatomidae, in the Asopinae subfamily (Schuh \& Slater 1995). Beside the great diversity and the relation of this family with agroecosystems, recent surveys of pentatomids in natural environments highlighted the potential of the group as indicators of human impact on the environment (Schmidt \& Barcellos 2007, Mendonça et al. 2009, Campos et al. 2009).

Buckup (1961) was the first to organize and list pentatomid species for Rio Grande do Sul State. Other surveys in distinct regions of Rio Grande do Sul, specially in agroecosystems, were published (i.e. Lopes et al. 1974, Gastal 1981, Gastal et al. 1981, Link \& Grazia 1983, 1987, Schmidt \& Barcellos 2007). For the Pampa biome the papers of Bonatto (1984), Lago \& Kaercher (1984), Barcellos (2006) and Mendonça et al. (2009) are fundamental references.

In this paper, the knowledge on Pentatomidae diversity of the Pampa biome is improved, based on surveys made in selected areas never collected before in the state of Rio Grande do Sul, Brazil. Identification of the Pentatomidae host plants were also one of the objectives of this paper, considering that only a few species have their host plants known.
The Serra do Sudeste region is characterized by an undulated relief, with altitudes varying from 200 to $500 \mathrm{~m}$. It sits on an old geological formation, the Precambrian granitic shield of the "Sul Riograndense" plateau (Boldrini 1997). The climate corresponds to Cfb of Köppen (Mota 1951). Annual median temperature varies from 16.3 to $17.7^{\circ} \mathrm{C}$ and the annual precipitation varies from 1370 to $1660 \mathrm{~mm}$, evenly distributed along the year (Boldrini 1997). The vegetation of the Serra do Sudeste studied areas is classified as grassland-forest mosaic (Teixeira et al. 1986, IBGE 2004, MMA 2007b, Cordeiro \& Hasenack 2009) where the former corresponds to the steppe type and the latter to the semideciduous seasonal forest.

This vegetation is still found in Parque de Espinilho da Barra do Quaraí (30 $11^{\prime}$ S and $57^{\circ} 30^{\prime} \mathrm{W}$ ) and surroundings, from the River Quaraí mouth to the Uruguayan border. This region represents the most significant part of the steppe-savanna in the pampas of Rio Grande do Sul; it also includes small patches of seasonal deciduous forests, composing the riparian forests. Climate conditions of the region are the most inhospitable of the state, with mean temperatures of $23.4{ }^{\circ} \mathrm{C}$, corresponding to Cfa of Köppen (Mota 1951), near the central Chaco where the rains are reduced to about $1300 \mathrm{~mm} / \mathrm{year}$ and are concentrated especially during summer (Mota 1951).

\section{Material and Methods}

Seven expeditions, from April, 2003 to April, 2004, were carried out to Canguçu and Caçapava do Sul, both counties located in the Serra do Sudeste region. During each expedition, four sites were sampled, two in Canguçu county (A1: $31^{\circ} 05^{\prime} 07^{\prime}$ ' $\mathrm{S}$ and $52^{\circ} 50^{\prime} 00^{\prime \prime} \mathrm{W}$; A2: $31^{\circ} 06^{\prime} \mathrm{S}$ and $52^{\circ} 52^{\prime} \mathrm{W}$ ), and two in Caçapava do Sul county (A3: $30^{\circ} 32^{\prime} 13^{\prime}$ ' $\mathrm{S}$ and $53^{\circ} 33^{\prime} 23^{\prime \prime} \mathrm{W}$ and $\mathrm{A} 4$ : $30^{\circ} 40^{\prime} \mathrm{S}$ and $53^{\circ} 27^{\prime} \mathrm{W}$ ).

In the westernmost region of RS (Barra do Quaraí county) four expeditions were carried out to ESP and surroundings from March, 2003 to January, 2004. Steppe-savannah sites were sampled, two representing the Espinilho formation (A5: $30^{\circ} 11^{\prime} \mathrm{S}$ and $57^{\circ} 30^{\prime} \mathrm{W}$ and $30^{\circ} 11^{\prime} \mathrm{S}$ and $57^{\circ} 35^{\prime} \mathrm{W}$ ), and two representing riparian forests (A6: $30^{\circ} 10^{\prime} 25^{\prime}$ ' $\mathrm{S}$ and $57^{\circ} 33^{\prime} \mathrm{W}$, $30^{\circ} 11^{\prime} 7^{\prime \prime} \mathrm{S}$ and $\left.57^{\circ} 35^{\prime} 50^{\prime \prime} \mathrm{W}\right)$.

Sweeping net (35 $\mathrm{cm}$ of diameter) was used to sample the herb layer in selected areas in order to cover all physiognomic characteristics of each site. Beating tray $\left(1 \mathrm{~m}^{2}\right)$ was used to sample trees and shrubs. Sampling included two people working for fixed periods of time (2 to 6 hours/area/expedition, Table 1). Visual inspection of the vegetation was also used for sampling. Whenever

Table 1. Pentatomidae sampling effort on each site in the Pampa biome, Rio Grande do Sul State, Brazil.

\begin{tabular}{|c|c|c|c|c|c|c|}
\hline $\begin{array}{c}\text { Sampling } \\
\text { site }^{*}\end{array}$ & $\begin{array}{l}\text { Sampling time/ } \\
\text { expedition } \\
\text { (hours) }\end{array}$ & \# collectors & $\begin{array}{l}\text { Sampling time/ } \\
\text { expedition/ } \\
\text { SN; BT ( hours) }\end{array}$ & $\begin{array}{c}\text { Total/time/ } \\
\text { expedition/ } \\
\text { SN; BT (hours) }\end{array}$ & Sampling date & $\begin{array}{c}\text { Total (timeXcollec } \\
\text { torXsampling) } \\
\text { (hours) }\end{array}$ \\
\hline A1 & 2 & 3 & $4 ; 2$ & $28 ; 14$ & \multirow{4}{*}{$\begin{array}{l}9-12 / \mathrm{IV} / 2003,12-16 / \\
\mathrm{VI} / 2003,7-11 / \mathrm{VIII} / 2003 \\
15-19 / \mathrm{X} / 2003,17-21 / \\
\mathrm{XII} / 2003,20-24 / \mathrm{II} / 2004 \\
30-3 / \mathrm{IV} / 2004\end{array}$} & 42 \\
\hline $\mathrm{A} 2$ & 4 & 3 & $8 ; 4$ & $56 ; 28$ & & 84 \\
\hline A3 & 2 & 3 & $4 ; 2$ & $28 ; 14$ & & 42 \\
\hline A4 & 2 & 3 & $4 ; 2$ & $28 ; 14$ & & 42 \\
\hline A5 & 6 & 2 & $6 ; 6$ & $24 ; 24$ & \multirow{2}{*}{$\begin{array}{l}21-25 / \mathrm{III} / 2003,15-18 / \\
\mathrm{V} / 2003,17-20 / \mathrm{IX} / 2003, \\
10-13 / \mathrm{I} / 2004\end{array}$} & 48 \\
\hline A6 & 6 & 2 & $6 ; 6$ & $24 ; 24$ & & 48 \\
\hline
\end{tabular}

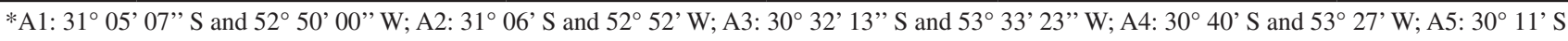
and $57^{\circ} 30^{\prime} \mathrm{W}, 30^{\circ} 11^{\prime} \mathrm{S}$ and $57^{\circ} 35^{\prime} \mathrm{W}$; A6: $30^{\circ} 10^{\prime} 25^{\prime}$ ' S and $57^{\circ} 33^{\prime} \mathrm{W}, 30^{\circ} 11^{\prime} 7^{\prime}$ ' $\mathrm{S}$ and $57^{\circ} 35^{\prime} 50^{\prime \prime} \mathrm{W}$; BT, Beating tray ;SN, Sweeping net. 
adults or nymphs were found feeding or adults housed in diapause were collected, the vegetation was considered as host plant.

Host plants were collected and herborized following Fidalgo \& Bononi (1989). Identification was based on the literature (Cabrera 1973, Troncoso 1978, Pilz \& Pereira 1988, Longhi-Wagner 2001, Mentz \& Oliveira 2004, Sobral et al. 2006). Vouchers specimens were deposited at Herbarium PEL, Departamento de Botânica, Instituto de Biologia, Universidade Federal de Pelotas.

Adults were slain in the field with killing jars and mounted in the laboratory. Identification was to species level whenever possible. The specimens were deposited in the collection of the Laboratório de Entomologia Sistemática, Departamento de Zoologia, Universidade Federal do Rio Grande do Sul (LES-UFRGS). The specimens which were not identified to species level, due to the present taxonomic knowledge of the taxon, were identified to genus level (Alveostethus Ruckes and Edessa Fabricius), and numbered according to the code adopted in the collection of LES-UFRGS for future comparisons and identification (see also Mendonça et al. 2009).

\section{Results and Discussion}

Fifty one species of Pentatomidae were collected, distributed in five subfamilies and 27 genera. The Pentatominae subfamily, which represents the greater diversity within the family (2.800 spp., Schuh \& Slater 1995), showed the greater number of species (32 spp.). All the registered localities represent new records to the local fauna (Table 2). Host plants were identified for nine pentatomid species (Table 3).

The genera with greater species richness were Euschistus ( 9 spp.), followed by Edessa (5 spp.), Chinavia (5 spp.) and Mormidea (5 spp.) (Table 2). These four genera combined correspond to $36.36 \%$ of the collected species. Among these genera, some species are recognized as important to agroecosystems in Rio Grande do Sul, i.e. Euschistus heros (Fabricius, 1798), Edessa meditabunda (Fabricius, 1784), Dichelops furcatus (Fabricius, 1775), Mormidea v-luteum (Lichtenstein, 1796) and M. notulifera Stål, 1860. The last two are associated with rice; $M$. notulifera was observed feeding on a native plant (Table 3). Also Piezodorus guildinii (Westwood, 1837), an important soybean pest in South America was observed feeding on a native plant in Canguçu county (Table 3 ).

The Pampa biome comprises a gamut of landscapes, and important steps are being taken to know their Pentatomidae fauna, although large areas remain unexplored. As far as we are aware, we add 22 species to the known fauna of the Brazilian Pampa biome.

Some of the available inventory sources cover environments distinct from the ones reported here. Bonatto (1984) sampled sites on grasslands, wetlands and vegetated dunes, in coastal ecosystems of the Pampa (Taim Ecological Station), with diverse methods as light traps, vacuum samplers and sweeping nets, however he only mentions 1063 pentatomids captured, without identification below family. Barcellos (2006) also sampled coastal environments, associated with the Patos lagoon, grasslands and low forests associated to sandy soils (restingas) and again with diverse methods as sweeping net, beating tray, leaf litter samples and manual search (diurnal and nocturnal). Overall the latter authors report 47 species of Pentatomidae, 18 of which are common to our findings.

Mendonça et al. (2009) sampled riparian forests within a matrix of grassland and pastures in Bagé, a part of the Campanha plains, in a rapid assessment employing beating tray only as method. They found 25 species of Pentatomidae, of which 18 are common to what we report here. Since Bagé is closer to the localities we sampled, a high number of species in common was expected.

Lago \& Kaercher (1984) covered a vast area: 20 municipalities (including Canguçu) of 4 micro-regions (Southeastern Hills,
Southeastern Slopes, Campanha and Coastal Plain), but there is no information on sampling methods; actually there is no mention whether insects were sampled by the authors or obtained from other sources, or even whether only agroecosystems were sampled. They also fail to mention sampling sites from which each species was obtained, so there is no useful geographical information available from this source. They listed 34 species within Pentatomidae (including one Phloeinae, treated as a subfamily), of which 14 can also be found in our list.

There is limited knowledge on this biome overall, which constitutes a problematic ecoregion in terms of conservation, given extensive economic use and small area protected by conservation units (Overbeck et al. 2007). There is also a paucity of past and present literature information on the biodiversity and biogeography of Pentatomidae as shown above. We therefore do not have a proper assessment of the pentatomid fauna of this region, and our powers to derive patterns of endemism, rarity, and thus to contribute to the conservation of the Pampa is still very limited. We expect the information provided here and a continuity of the exploration of this region to generate a clearer view of this group, so as to fulfill the Pentatomidae role as an ecological indicator (as suggested by Brown 1997 for the Atlantic Forest) and expand it to the Pampa.

List of the species, in the subfamilies (see Table 2 for presence and abundance of the species in distinct sites):

\section{ASOPINAE AMYOT \& SERVILLE}

1. Brontocoris tabidus (Signoret, 1863)

2. Podisus nigrispinus (Dallas, 1851)

3. Supputius cincticeps (Stål, 1860)

4. Tylospilus cloelia (Stål, 1862)

5. Tynacantha marginata (Dallas, 1851)

\section{CYRTOCORINAE DISTANT}

6. Cyrtocoris gibbus (Fabricius, 1803)

\section{DISCOCEPHALINAE FIEBER}

7. Alveostethus $\mathrm{sp} .1$

8. Antiteuchus tripterus (Fabricius, 1787)

9. Dinocoris lineatus (Dallas, 1852)

\section{EDESSINAE KIRKALDY}

10. Brachystethus geniculatus (Fabricius, 1787)

11. Edessa albomarginata (Stål, 1855)

12. Edessa meditabunda (Fabricius, 1784)

13. Edessa rufomarginata (De Geer, 1773)

14. Edessa scabriventris Stål, 1860

15. Edessa sp. 2

16. Edessa sp. 3

17. Edessa sp.4

18. Edessa sp.5 
Bunde, P.R.S. et al.

Table 2. Abundance of Pentatomidae species collected in the surveyed areas of the Pampa biome, Rio Grande do Sul State, Brazil.

\begin{tabular}{|c|c|c|c|c|c|c|c|c|c|}
\hline Subfamily & Species & A1 & $\mathbf{A 2}$ & $\mathbf{A 3}$ & A4 & A5 & A6 & Total & Sampling \\
\hline \multirow[t]{5}{*}{ Asopinae } & Brontocoris tabidus (Signoret, 1863) & 0 & 0 & 0 & 0 & 0 & 3 & 3 & BT \\
\hline & Podisus nigrispinus (Dallas, 1851) & 0 & 0 & 1 & 0 & 1 & 5 & 7 & BT \\
\hline & Supputius cincticeps (Stål, 1860) & 2 & 0 & 0 & 0 & 0 & 1 & 3 & BT \\
\hline & Tylospilus cloelia (Stål, 1862) & 0 & 0 & 0 & 0 & 0 & 2 & 2 & BT \\
\hline & Tynacantha marginata (Dallas, 1851) & 0 & 0 & 0 & 0 & 0 & 3 & 3 & BT \\
\hline Cyrtocorinae & Cyrtocoris gibbus (Fabricius, 1803) & 0 & 0 & 0 & 0 & 0 & 7 & 7 & $\mathrm{BT}$ \\
\hline \multirow[t]{3}{*}{ Discocephalinae } & Alveostethus sp.1 & 0 & 0 & 0 & 0 & 0 & 4 & 4 & BT \\
\hline & Antiteuchus tripterus (Fabricius, 1787) & 0 & 0 & 0 & 0 & 0 & 14 & 14 & BT \\
\hline & Dinocoris lineatus (Dallas, 1852) & 0 & 0 & 0 & 0 & 0 & 4 & 4 & BT \\
\hline \multirow[t]{10}{*}{ Edessinae } & Brachystethus geniculatus (Fabricius, 1787) & 3 & 2 & 3 & 0 & 0 & 2 & 10 & BT; SN \\
\hline & Edessa albomarginata (Stål, 1855) & 0 & 0 & 0 & 0 & 2 & 0 & 2 & BT \\
\hline & E. meditabunda (Fabricius, 1784) & 0 & 4 & 0 & 3 & 5 & 6 & 18 & $\mathrm{SN}$ \\
\hline & E. rufomarginata (De Geer, 1773) & 3 & 0 & 2 & 0 & 0 & 3 & 8 & BT \\
\hline & E. scabriventris Stål, 1860 & 0 & 0 & 1 & 0 & 0 & 0 & 1 & BT; SN \\
\hline & Edessa sp. 2 & 0 & 0 & 0 & 2 & 0 & 10 & 12 & BT \\
\hline & Edessa sp. 3 & 5 & 4 & 1 & 0 & 0 & 0 & 10 & $\mathrm{BT}$ \\
\hline & Edessa sp.4 & 0 & 0 & 0 & 0 & 0 & 1 & 1 & BT \\
\hline & Edessa sp.5 & 0 & 0 & 0 & 0 & 1 & 6 & 7 & $\mathrm{BT}$ \\
\hline & Olbia caprina (Stål, 1862) & 0 & 0 & 0 & 0 & 0 & 1 & 1 & $\mathrm{BT}$ \\
\hline \multirow[t]{32}{*}{ Pentatominae } & Acledra bonariensis (Stål, 1859) & 3 & 2 & 0 & 0 & 0 & 0 & 5 & SN \\
\hline & A. kinbergii (Stål, 1859) & 0 & 0 & 0 & 0 & 5 & 0 & 5 & BT \\
\hline & Arvelius albopunctatus (De Geer, 1773) & 1 & 11 & 0 & 0 & 2 & 1 & 15 & $\mathrm{SN}$ \\
\hline & Chinavia aseada (Rolston, 1983) & 0 & 2 & 0 & 3 & 0 & 0 & 5 & SN \\
\hline & Chinavia erythrocnemis (Berg, 1878) & 0 & 0 & 0 & 0 & 0 & 9 & 9 & BT \\
\hline & Chinavia longicorialis (Breddin, 1901) & 1 & 10 & 0 & 1 & 4 & 0 & 16 & BT; SN \\
\hline & Chinavia musiva (Berg, 1878) & 0 & 0 & 0 & 0 & 2 & 0 & 2 & BT \\
\hline & Chinavia nigridorsata (Breddin, 1901) & 0 & 0 & 0 & 0 & 2 & 1 & 3 & BT; SN \\
\hline & Chlorocoris complanatus (Guérin-Ménéville, 1831) & 0 & 0 & 0 & 0 & 0 & 3 & 3 & BT \\
\hline & Chloropepla vigens (Stål, 1860) & 3 & 1 & 0 & 0 & 0 & 0 & 4 & BT \\
\hline & Dichelops furcatus (Fabricius, 1775) & 11 & 5 & 12 & 3 & 3 & 4 & 38 & $\mathrm{SN}$ \\
\hline & Euschistus aceratos Berg, 1894 & 0 & 0 & 1 & 1 & 0 & 0 & 2 & $\mathrm{SN}$ \\
\hline & Euschistus circumfusus Berg, 1883 & 1 & 0 & 0 & 0 & 0 & 0 & 1 & SN \\
\hline & Euschistus cornutus Dallas, 1851 & 0 & 0 & 1 & 0 & 4 & 2 & 7 & $\mathrm{SN}$ \\
\hline & Euschistus heros (Fabricius, 1798) & 0 & 0 & 1 & 0 & 0 & 0 & 1 & $\mathrm{SN}$ \\
\hline & Euschistus irroratus Bunde, Grazia \& Mendonça Jr., 2006 & 0 & 7 & 0 & 0 & 0 & 0 & 7 & $\mathrm{BT}$ \\
\hline & Euschistus paranticus Grazia, 1987 & 5 & 6 & 0 & 0 & 1 & 0 & 12 & $\mathrm{SN}$ \\
\hline & Euschistus picticornis Stål, 1872 & 0 & 1 & 0 & 1 & 0 & 0 & 2 & $\mathrm{SN}$ \\
\hline & Euschistus sharpi Bergroth, 1891 & 0 & 1 & 0 & 0 & 0 & 0 & 1 & $\mathrm{SN}$ \\
\hline & Grazia tincta (Distant, 1890) & 0 & 0 & 0 & 0 & 1 & 0 & 1 & BT \\
\hline & Loxa deducta Walker, 1867 & 1 & 2 & 2 & 0 & 2 & 2 & 9 & BT \\
\hline & Mayrinia curvidens (Mayr, 1864) & 0 & 0 & 0 & 0 & 0 & 4 & 4 & BT \\
\hline & Mormidea cornicollis Stål, 1860 & 1 & 12 & 3 & 0 & 0 & 0 & 16 & $\mathrm{SN}$ \\
\hline & M. notulifera Stål, 1860 & 15 & 3 & 0 & 1 & 0 & 0 & 19 & $\mathrm{SN}$ \\
\hline & M. paupercula Berg, 1879 & 10 & 4 & 8 & 4 & 36 & 9 & 71 & SN \\
\hline & M. v-luteum (Lichtenstein, 1796) & 0 & 4 & 2 & 13 & 11 & 12 & 42 & $\mathrm{SN}$ \\
\hline & Nezara viridula (Linnaeus, 1798) & 0 & 3 & 0 & 0 & 0 & 0 & 3 & SN \\
\hline & Oebalus poecilus (Dallas, 1851) & 0 & 0 & 0 & 0 & 0 & 1 & 1 & SN \\
\hline & O. ypsilongriseus (De Geer, 1773) & 0 & 0 & 1 & 1 & 5 & 16 & 23 & SN \\
\hline & Piezodorus guildinii (Westwood, 1837) & 0 & 2 & 2 & 0 & 1 & 2 & 6 & SN \\
\hline & Thyanta humilis Bergroth, 1891 & 4 & 20 & 11 & 4 & 0 & 14 & 53 & SN \\
\hline & Thyanta perditor (Fabricius, 1794) & 0 & 0 & 1 & 0 & 0 & 0 & 1 & $\mathrm{SN}$ \\
\hline Total & & 69 & 106 & 53 & 37 & 88 & 152 & 505 & \\
\hline
\end{tabular}

*A1: $31^{\circ} 05^{\prime} 07^{\prime \prime} \mathrm{S}$ and $52^{\circ} 50^{\prime} 00^{\prime \prime} \mathrm{W}$; A2: $31^{\circ} 06^{\prime} \mathrm{S}$ and $52^{\circ} 52^{\prime} \mathrm{W}$; A3: $30^{\circ} 32^{\prime} 13^{\prime \prime} \mathrm{S}$ and $53^{\circ} 33^{\prime} 23^{\prime \prime} \mathrm{W}$; A4: $30^{\circ} 40^{\prime} \mathrm{S}$ and $53^{\circ} 27^{\prime} \mathrm{W}$; A5: $30^{\circ} 11^{\prime} \mathrm{S}$ and $57^{\circ} 30^{\prime} \mathrm{W}, 30^{\circ} 11^{\prime} \mathrm{S}$ and $57^{\circ} 35^{\prime} \mathrm{W}$; A6: $30^{\circ} 10^{\prime} 25^{\prime \prime} \mathrm{S}$ and $57^{\circ} 33^{\prime} \mathrm{W}, 30^{\circ} 11^{\prime} 7^{\prime \prime} \mathrm{S}$ and $57^{\circ} 35^{\prime}$ 50” W; BT, Beating tray; SN, Sweeping net. 
Table 3. Host plants (families and species) of Pentatomidae species collected in Serra do Sudeste, Rio Grande do Sul State, Brazil.

\begin{tabular}{|c|c|c|c|}
\hline $\begin{array}{c}\text { Host plants } \\
\text { families }\end{array}$ & Host species & Pentatomidae species $^{1}$ & Voucher $^{2}$ \\
\hline \multirow[t]{3}{*}{ Anacardiaceae } & Lithraea sp. & 10,22 & P.R.S.Bunde s.n. (PEL24506) \\
\hline & Schinus lentiscifolius Marchand & 25 & E.J.E.Silva s.n. (PEL 24507) \\
\hline & Schinus polygamus (Cav.) Cabrera & $13,25,40,51$ & P.R.S.Bunde s.n. (PEL 24508, PEL 24509) \\
\hline \multirow[t]{2}{*}{ Asteraceae } & Gochnatia polymorpha (Less.) Cabrera & 50 & E.J.E.Silva s.n. (PEL 24510) \\
\hline & Trixis praestans (Vell.) Cabrera & 50 & P.R.S.Bunde s.n. (PEL 24511) \\
\hline Cannabaceae & Celtis ehrenbergiana (Klotzsch) Liebm. & & P.R.S.Bunde s.n. (PEL 24512) \\
\hline Euphorbiaceae & Sebastiania brasiliensis Spreng. & & P.R.S.Bunde s.n. (PEL 24513) \\
\hline Myrtaceae & Eugenia uniflora L. & 13 & P.R.S.Bunde s.n. (PEL 24514) \\
\hline Poaceae & Pseudechinolaena polystachya (Kunth) Stapf & 22,43 & E.J.E.Silva s.n. (PEL 24515) \\
\hline Polygonaceae & Polygonum punctatum Elliott & & E.J.E.Silva s.n. (PEL 24516) \\
\hline \multirow[t]{3}{*}{ Solanaceae } & Solanum paniculatum $\mathrm{L}$. & 13 & E.J.E.Silva s.n. (PEL 24517) \\
\hline & Solanum pseudoaculeatissimum L.A. Mentz & 22 & E.J.E.Silva s.n. (PEL 24518) \\
\hline & Solanum viarum Dunal & 22,49 & P.R.S.Bunde s.n. (PEL 24519) \\
\hline Verbenaceae & Aloysia gratissima (Gillies \& Hook.) Tronc. & 10 & P.R.S.Bunde s.n. (PEL 24520) \\
\hline
\end{tabular}

${ }^{1}$ The number corresponds to the species as shown in the species list (p. 3-5); ${ }^{2}$ Voucher number in the Herbarium PEL, Departamento de Botânica of Instituto de Biologia in Universidade Federal de Pelotas.

19. Olbia caprina (Stål, 1862)

\section{PENTATOMINAE LEACH}

20. Acledra bonariensis (Stål, 1859)

21. Acledra kinbergii (Stål, 1859)

22. Arvelius albopunctatus (De Geer, 1773)

23. Chinavia aseada (Rolston, 1983)

24. Chinavia erythrocnemis (Berg, 1878)

25. Chinavia longicorialis (Breddin, 1901)

26. Chinavia musiva (Berg, 1878)

27. Chinavia nigridorsata (Breddin, 1901)

28. Chlorocoris complanatus (Guérin-Menevile, 1831)

29. Chloropepla vigens (Stål, 1860)

30. Dichelops furcatus (Fabricius, 1775)

31. Euschistus aceratos Berg, 1894

32. Euschistus circumfusus Berg, 1883

33. Euschistus cornutus Dallas, 1851

34. Euschistus heros (Fabricius, 1798)

35. Euschistus irroratus Bunde, Grazia \& Mendoça Jr., 2006

36. Euschistus paranticus Grazia, 1987

37. Euschistus picticornis Stål, 1872

38. Euschistus sharpi Bergroth, 1891

39. Grazia tincta (Distant, 1890)
40. Loxa deducta Walker, 1867

41. Mayrinia curvidens (Mayr, 1864)

42. Mormidea cornicollis Stål, 1860

43. Mormidea notulifera Stål, 1860

44. Mormidea paupercula Berg, 1879

45. Mormidea v-luteum (Lichtenstein, 1796)

46. Nezara viridula (Linnaeus, 1758)

47. Oebalus poecilus (Dallas, 1851)

48. Oebalus ypsilongriseus (De Geer, 1773)

49. Piezodorus guildinii (Westwood, 1837)

50. Thyanta humilis Bergroth, 1891

51. Thyanta perditor (Fabricius, 1794)

\section{References}

AGUIAR, A.P., SANTOS, B.F., COURI, M.S., RAFAEL, J.A., COSTA, C., IDE, S., DUARTE, M., GRAZIA, J., SCHWERTNER, C.F., FREITAS, A.V.L. \& AZEVEDO, C.O. 2009. Insecta. In Estado da arte e perspectivas para a Zoologia no Brasil (R.M. Rocha \& W.A. Boeger, orgs.) Ed. UFPR, Curitiba, p. 131-155.

BARCELLOS, A. 2006. Hemípteros terrestres. In Biodiversidade 25: Região da Lagoa do Casamento e dos Butiazais de Tapes, Planície Costeira do Rio Grande do Sul (F.G. Becker, R.A. Ramos \& L.A. Moura, orgs.). Ministério do Meio Ambiente, Brasília, p. 198-209. http://www.mma.gov.br

BOLDRINI, I.I. 1997. Campos do Rio Grande do Sul: caracterização fisionômica e problemática ocupacional. Bol. Inst. Biocienc. Univ. Fed. Rio Gd. Sul 56:1-39.

BONATTO, S.L. 1984. Resultados preliminares do levantamento da entomofauna da Estação Ecológica do Taim, Rio Grande do Sul, Brasil. In Semana Universitária Gaúcha de Debates Ecológicos, 21aㄹ 22a, 23 , $24^{\mathrm{a}}$. Anais. Universidade Federal do Rio Grande do Sul, Porto Alegre, p. $46-54$. 
Bunde, P.R.S. et al.

BROWN Jr., K.S. 1997. Diversity, disturbance, and sustainable use of Neotropical forests: insects as indicators for conservation monitoring. J. Insect. Conserv. 1:25-42.

BUCKUP, L. 1961. Os pentatomídeos do Estado do Rio Grande do Sul (Brasil) (Hemiptera-Heteroptera-Pentatomidae). Iheringia, Sér. Zool. 16:1-24.

BUNDE, P.R.S., GRAZIA, J. \& MENDONÇA FILHO, M. 2006. Nova espécie de Euschistus (Mitripus) da Argentina e sul do Brasil (Hemiptera, Pentatomidae, Pentatominae). Iheringia, Sér. Zool. 96(3):289-291.

CABRERA, A.L. 1973. Compositae. In Flora Ilustrada de Entre Rios (Argentina). pt. 6. (A. Burkart, org.). INTA, Buenos Aires, p. 106-554.

CAMPOS, L.A., BERTOLIN, T.B.P., TEIXEIRA, R.A. \& MARTINS, F.S. 2009. Diversidade de Pentatomoidea (Hemiptera, Heteroptera) em três fragmentos de Mata Atlântica no sul de Santa Catarina. Iheringia, Sér. Zool. 99(2):165-171.

FERRER, J., MALABARBA, L.R. \& COSTA, W.J.E.M. 2008. Austrolebias paucisquama (Cyprinodontiformes: Rivulidae), a new species of annual killifish from southern Brazil. Neotrop. Ichthyol. 6(2):175-180.

FIDALGO, O. \& BONONI, V.L.R. 1989. Técnica de coleta, preservação e herborização de material botânico. São Paulo. Série Documentos.

GASTAL, H.A. O., LANZER-DE-SOUZA, M.E. \& GALILEO, M.H.M. 1981. Diversidade e similaridade de comunidades de Pentatomidae (Hemiptera) capturados com armadilha luminosa na Grande Porto Alegre, Rio Grande do Sul. Iheringia, Sér. Zool. 59:5-12.

GASTAL, H.A.O. 1981. Lista Preliminar dos asopíneos do Estado do Rio Grande do Sul, Brasil (Insecta, Hemiptera, Pentatomidae). Iheringia, Sér. Zool. 57:119-127.

GRAZIA, J. \& SCHWERTNER, C.F. 2008. Pentatomidae e Cyrtocoridae. In Biodiversidad de Artrópodos Argentinos (L.E. Claps, G. Debandi \& S. Roig-Juñent, dirs.) Sociedad Entomologica Argentina, Tucumán, v. 2, p. 223-234.

GRAZIA, J., CAVICCHIOLI, R.R., WOLFF, V.R.S., FERNANDES, J.A.M \& TAKIYA, D.A. in press. Hemiptera. In Insetos do Brasil: Diversidade e Taxonomia. (J.A. Rafael, G.A.R. Melo, C.J.B. Carvalho, S. Casari \& R. Constantino, orgs.).

INSTITUTO BRASILEIRO DE GEOGRAFIA E ESTATÍSTICA - IBGE. 2004. Mapa da vegetação do Brasil e Mapa de Biomas do Brasil. http:// www.ibge.gov.br

LAGO, I.C.S. \& KAERCHER, F. 1984. Hemípteros de ocorrência nas regiões Encosta do Sudeste, Serra do Sudeste, Campanha e Litoral do Rio Grande do Sul. Agros 19:92-103.

LEVINSOHN, T.M. \& PRADO, P.K.S. 2002. Biodiversidade Brasileira. Síntese do estado atual do conhecimento. Editora Contexto Acadêmico, São Paulo.

LEWINSOHN, T.M., PRADO, P.K.L. \& ALMEIDA, A.M. 2001. Inventários Bióticos Centrados em recursos: Insetos fitófagos e plantas hospedeiras. In Conservação da biodiversidade em ecossistemas tropicais. Avanços conceituais e revisão de novas metodologias de avaliação e monitoramento (B.F.S. Dias \& I. Garay, eds.) Vozes, Petrópolis.

LINK, D. \& GRAZIA, J. 1983. Pentatomídeos capturados em armadilha luminosa, em Santa Maria, RS, Brasil. An. Soc. ent. Brasil 12(1):123-125.

LINK, D. \& GRAZIA, J. 1987. Pentatomídeos da região central do Rio Grande do Sul (Heteroptera). An. Soc. ent. Brasil 16(1):115-129.
LONGHI-WAGNER, H.M. 2001. Gênero Pseudechinolaena. In Flora fanerogâmica do Estado de São Paulo- Poaceae, v. 1. (H.M. LonghiWagner, V. Bittrich, M.G.L. Wanderley \& G. Shepherd, orgs). Hucitec, São Paulo, p. 231-232.

LOPES, O.J., LINK, D. \& BASSO, I.V. 1974. Pentatomídeos de Santa Maria - lista preliminar de plantas hospedeiras. Rev. Centr. Ciênc. Rur. Santa Maria 4:317-322.

MENDONÇA Jr., M.S., SCHWERTNER, C.F. \& GRAZIA, J. 2009. Diversity of Pentatomoidea (Hemiptera) in riparian forests of Bagé, RS, southern Brazil: taller forests, more bugs. Rev. Bras. Entomol. 53(1):121-127.

MENTZ, L.A. \& OLIVEIRA, P.L. 2004. Solanum (Solanaceae) na Região Sul do Brasil. Pesqui., Bot. 54:1-327.

MINISTÉRIO DO MEIO AMBIENTE - MMA. 2007a. Áreas Prioritárias para a Conservação, Uso sustentável e Repartição dos Benefícios da Biodiversidade Brasileira. Ministério do Meio Ambiente, Brasília. http:// www.mma.gov.br/portalbio

MINISTÉRIO DO MEIO AMBIENTE - MMA. 2007b. Mapas de Cobertura Vegetal dos Biomas Brasileiros: relatório Bioma Pampa. http://www. mma.gov.br/portalbio

MOTA, F.S. 1951. Estudos do clima do Estado do Rio Grande do Sul, segundo o sistema de W. Köppen. Rev. Bras. Geogr. 13(2):275-284.

OVERBECK, G.E., MULLER, S.C., FIDELIS, A., PFADENHAUER, J., PILLAR, V.D., BLANCO, C.C., BOLDRINI, I.I., BOTH, R. \& FORNECK, E.D. 2007. Brazil's neglected biome: The South Brazilian Campos. Perspectives Plant Ecol. Evol. Syst. 9:101-116.

PANIZZI, A.R., MCPHERSON, J.E., JAMES, D.J., JAVAHERY, M. \& MCPHERSON, R.M. 2000. Stink bugs (Pentatomidae). In Heteroptera of economic importance (C.W. Schaefer \& A.R. Panizzi, eds.). CRC Press, Boca Raton, p. 421-474.

PILZ, A.B.F. \& PEREIRA, A.B. 1988. O gênero Polygonum L. no Rio Grande do Sul. Pesqui., Bot. 38:43-72.

SCHMIDT, L.S. \& BARCELLOS, A. 2007. Abundância e riqueza de espécies de Heteroptera (Hemiptera) do Parque Estadual do Turvo, sul do Brasil: Pentatomoidea. Iheringia, Sér. Zool. 97:73-79.

SCHUH, T.R. \& SLATER, J.A. 1995. True bugs of the World (Hemiptera: Heteroptera). Classification and natural history. Cornell University, Ithaca.

SOBRAL, M., JARENKOW, J.A., BRACK, P., IRGANG, B.E., LAROCCA, J. \& RODRIGUES, R.S. 2006. Flora arbórea e arborescente do Rio Grande do Sul, Brasil. v. 1. RiMa/Novo Ambiente, São Carlos.

TEIXEIRA, M.B., COURA NETO, A.B., PASTORE, U. \& RANGEL FILHO, L.R. 1986. Vegetação: as regiões fitoecológicas, sua natureza e seus recursos econômicos; estudo fitogeográfico. In Levantamento de recursos naturais. IBGE, Rio de Janeiro, p. 541-620.

TRONCOSO, N.S. 1978. Verbenaceae. In Flora Ilustrada de Entre Rios, pt. 5. (A. Burkart, org.). INTA, Buenos Aires, p. 229-294.

VERRASTRO, L., VERONESE, L., BUJES, C.S. \& DIAS FILHO, M.M. 2003. A new species of Liolaemus from southern Brazil (Iguania, Tropiduridae). Herpetologica 59(1):252-277.

Received 25/03/2010

Revised 23/06/2010

Accepted 12/07/2010 